\title{
Survivorship Bias Following Military Thoracic Injuries: Reply
}

\author{
J. J. Morrison · M. J. Midwinter · J. O. Jansen
}

Published online: 28 September 2011

(C) Société Internationale de Chirurgie 2011

We are grateful to Dr. Calvin and co-workers for their interest in our article [1] and the analysis of their patient population. As they clearly demonstrate, survivorship bias is an important issue when comparing the casualty load from a Role 3 facility (deployed Field Hospital, often in an austere environment) to a Role 4 facility (mature hospital outwith an operational theater, with subspecialty representation).

However, there are several other factors that are important to recognize in this analysis. Our examination was of consecutive admissions to the Field Hospital, and the majority of patients in our series were Afghan Nationals, a group not represented in an analysis of a Role 4 population. This patient group tends to sustain a penetrating injury pattern as the torso is generally not protected by body armor. In contrast, U.K. soldiers in Afghanistan tend to sustain blast-related thoracic injury, as demonstrated by Dr. Calvin's analysis. Our article focused specifically on the management of penetrating injury.
It was interesting to read that no patients required a thoracotomy at the Role 4 facility. We certainly agree with Dr. Calvin that emergency thoracotomy, applied judiciously in the correct patient group, can yield unexpected survivors. Indeed, we reported two unexpected survivors in our study [1].

I believe this dialogue best demonstrates the need for a joined-up evaluation of wartime thoracic injury from the point-of-wounding to discharge at a Role 4 hospital.

\section{References}

1. Morrison J, Midwinter M, Jansen J (2011) Ballistic thoracoabdominal injury: analysis of recent military experience in Afghanistan. World J Surg 35:1396-1401. doi:10.1007/s00268-011-1046-2
J. J. Morrison $(\bowtie) \cdot$ M. J. Midwinter

Academic Department of Military Surgery and Trauma, Royal

Centre for Defence Medicine, Birmingham Research Park,

Vincent Drive, Birmingham, Edgbaston B15 2SQ, UK

e-mail: jonny_morrison@doctors.org.uk

J. O. Jansen

Royal Army Medical Corps, 144 Parachute Medical Squadron,

2 Priory Road, Hornsey, London N8 7RD, UK 